\title{
GERENCIAMENTO DE PRODUTOS QUÍMICOS NA GDU*
}

\author{
Thiago Guimarães Teixeira ${ }^{1}$ \\ Carlos Henrique Gomes ${ }^{1}$ \\ Emerson Alexandre Capchek ${ }^{1}$ \\ Ítalo Pereira Gomes de Almeida ${ }^{2}$
}

\section{Resumo}

Produtos químicos são os ingredientes essenciais para os processos de tratamento de águas e efluentes. A GDU consome mais de 30 produtos químicos diferentes utilizados em suas 17 estações de tratamento de água e efluentes distribuídas em toda a Usina Presidente Vargas (UPV). A gestão dos estoques dos produtos químicos, acompanhada do consumo padrão de cada um, contribuem para a garantia da qualidade da água tratada e no custo de tratamento de cada ETE. Por outro lado, a falta ou a deficiência de um sistema de gerenciamento acarreta em descontroles dos parâmetros de qualidade da água e aumenta custos por imprevisibilidades no processo, podendo inclusive causar danos aos processos clientes. O grupo em conjunto implantou um sistema de gerenciamento visual dos estoques de produtos químicos em cada área de sua responsabilidade, pois foi evidenciado muitas ocorrências de descontrole da qualidade da água devido à falta de produtos químicos na área, e como consequência, elevados pedidos de produtos químicos em emergência. A principal ação tomada para a implantação desse sistema de gerenciamento de produtos químicos foi estabelecer, por meio de gráficos de controle, um sistema de gerenciamento visual, permitindo conhecer, acompanhar e prever o consumo e data de novos pedidos. Dentre os benefícios da implantação deste trabalho, podemos citar: a) Efetivação dos pedidos de produtos químicos com antecedência; b) Entregas programadas na data de necessidade, com estabelecimento de nível de estoque de segurança de produto nas áreas; c) Redução do número de solicitações de produtos químicos em emergências; d) Envolvimento dos operadores nos consumos e controles de estoques de produtos químicos, através do sistema de gerenciamento; e) Parâmetros de qualidade da água estabilizados; f) Redução do risco de interferência nos clientes por má qualidade da água; g) Redução de desperdícios; h) Custos evitados com transportes e multas ambientais.

Palavras-chave: Gerenciamento; Produtos químicos; Estoque.

\begin{abstract}
Chemical products are essential ingredients for water and wastewater treatment processes. The GDU consumes more than 30 different chemicals used in its 17 water treatment plants and wastewater distributed throughout the Presidente Vargas Plant (UPV). The management of stockpiles of chemicals, accompanied by the standard of each consumer, contribute to ensuring the treated water quality and the cost of treating each WWTP (Wastewater Treatment Plant). On the other hand, the lack or deficiency of a management system leads to upsets of water quality parameters and increases costs for unpredictability in the process and may even cause damage to client processes. The group jointly implemented a visual management system of chemical stocks in every area of its responsibility, as evidenced many instances of lack of water quality due to lack of chemicals in the area, and consequently, higher product orders chemical emergency. The main action taken for the implementation of this chemicals management system was to establish through control charts, a visual management system, allowing knowing, monitoring and predicting the consumption and date of order. Among the benefits of implementing this work include: a) Effective applications for chemicals in advance; b) deliveries scheduled at the time of need, with inventory levels to establish product safety areas; c) Reduction of the number of requests of chemical in emergencies; d) Involvement of operators in consumption and inventory controls of chemicals, through the management system of chemicals; e) Stabilized water quality parameters; f) Reduction of the risk of interference with customers for poor water quality; g ) waste reduction; h) costs avoided with transport and environmental fines.
\end{abstract}

CHEMICALS MANAGEMENT IN GDU

Keywords: Management; Chemicals; Stock.

1 Supervisor de Águas e Efluentes da Gerência de Utilidades, Companhia Siderúrgica Nacional (CSN), Volta Redonda, Rio de Janeiro-RJ, Brasil.

2 Líder de Águas e Efluentes da Gerência de Utilidades, CSN, Volta Redonda, Rio de Janeiro-RJ, Brasil. 


\section{INTRODUÇÃO}

O atual cenário vivido pelas organizações obriga a qualquer gestor a controlar mais de perto o que acontece em seu processo. No caso de tratamento de efluentes, temos os produtos químicos, que são os ingredientes básicos e essenciais para se garantir os resultados esperados em qualidade da água, e que contribuem significativamente com a parcela de custo da empresa.

A GDU (Gerência de Distribuição de Utilidades) consome mais de 30 produtos químicos diferentes, utilizados em suas 17 estações de tratamento de água e efluentes distribuídas em toda a Usina Presidente Vargas (UPV).

A gestão dos estoques dos produtos químicos, acompanhada do consumo padrão de cada um, contribuem para a garantia da qualidade da água tratada e no custo de tratamento de cada ETE. Por outro lado, a falta ou a deficiência de um sistema de gerenciamento acarreta em descontroles dos parâmetros de qualidade da água e aumenta custos por imprevisibilidades no processo, podendo inclusive causar danos aos processos clientes e meio ambiente.

O presente grupo se reuniu com o objetivo de implantar um sistema de gerenciamento dos produtos químicos das áreas de sua responsabilidade, pois foi evidenciado um descontrole dos estoques, faltando produtos nas áreas, elevados pedidos de produtos químicos em emergência, e prejudicando o parâmetro de qualidade da água.

A figura 1 a seguir ilustra como era feito o controle anteriormente, mostrando falhas no acompanhamento e pouca informação gerencial.

\begin{tabular}{|c|c|c|c|c|c|c|c|}
\hline \multicolumn{8}{|c|}{$\begin{array}{l}\text { PLANILHA DE CONTROLE DE DOSAGEM DE PRODUTO QUIMIICO (por litro) } \\
\text { PAC } 47503\end{array}$} \\
\hline DIA & Estoque Anterior & Consumo T1 & Consumo T2 & Consumo T3 & Consumo T4 & Recebimento & Estoque Atual \\
\hline 1 & 210 & 30 & 0 & 50 & 0 & & 130 \\
\hline 2 & 130 & 0 & 70 & 0 & 0 & 1000 & 1060 \\
\hline 3 & 1060 & 110 & 30 & 20 & 60 & & 840 \\
\hline 4 & 840 & 45 & 30 & 20 & 20 & & 725 \\
\hline 5 & 725 & 25 & 0 & 20 & 30 & & 650 \\
\hline 6 & 650 & 20 & 30 & 20 & 20 & & 560 \\
\hline 7 & 560 & 20 & 20 & 20 & 20 & & 480 \\
\hline 8 & 480 & 20 & 0 & 10 & 20 & & 430 \\
\hline 9 & 430 & 10 & 10 & 70 & 30 & 1000 & 1310 \\
\hline 10 & 1310 & 10 & 30 & & 10 & & 1260 \\
\hline 11 & 1260 & 20 & 10 & & 50 & & 1180 \\
\hline 12 & 1180 & 20 & 10 & 20 & 0 & & 1130 \\
\hline 13 & 1130 & 20 & 20 & 10 & 50 & & 1030 \\
\hline 14 & 1030 & 0 & 20 & 20 & 20 & & 970 \\
\hline 15 & 970 & 10 & 10 & 10 & 20 & & 920 \\
\hline 16 & 920 & 20 & 20 & 0 & 20 & & 860 \\
\hline 17 & 860 & 20 & 10 & 40 & 10 & & 780 \\
\hline 18 & 780 & 10 & & 10 & 20 & & 740 \\
\hline 19 & 740 & 10 & & 20 & 20 & & 690 \\
\hline 20 & 690 & 20 & 20 & 0 & 20 & & 630 \\
\hline 21 & 630 & 10 & & 0 & 0 & & 620 \\
\hline 22 & 620 & 10 & 0 & 0 & 0 & & 610 \\
\hline 23 & 610 & 0 & & 10 & 0 & & 600 \\
\hline 24 & 600 & 10 & 10 & 10 & 10 & & 560 \\
\hline 25 & 560 & 0 & 10 & 10 & 0 & & 540 \\
\hline 26 & 540 & 0 & 0 & 0 & 0 & & 540 \\
\hline
\end{tabular}

Figura 1: Exemplo do controle anterior - falta de acompanhamento em alguns turnos e pouca informação gerencial. Fonte: GDU

\section{MATERIAIS E MÉTODOS}

Para a implantação do sistema de gerenciamento dos produtos químicos na GDU, foi necessário basicamente criar um sistema de acompanhamento visual, por meio de gráficos de controle, que permitiu a visualização do estoque, o consumo, o estoque de segurança, e a tendência de quando o produto iria acabar. Permitiu também ao gestor, fazer os pedidos dos produtos químicos com antecedência, evitando a falta deste insumo na área. 
As áreas envolvidas neste trabalho foram:

ETE-LG e ETE-ES-LG (trabalho piloto)

ETEO

ETE-LTQ\#2

ETE-MCC\#4

ETE-RH

ETE-CC

ETE-LD

ETE-AF\#2

ETE-AF\#3

ETE-TRT

CBR\#4

CBR\#14

CBR\#18

CBR\#20

CBR\#25

CBR\#26

Para a implantação do sistema de gerenciamento, foi necessário conhecer:

1 - Quais eram os produtos químicos aplicados nas áreas;

2 - Quanto tempo levava para que o produto fosse consumido;

3 - Qual era o tempo de entrega para fazer a colocação do pedido;

4 - Qual seria o nível de estoque de segurança que deveria ser estabelecido até a chegada do produto.

Após todas essas informações levantadas, foram criadas as planilhas de acompanhamento e plotados os gráficos de controle para acompanhamento pelos Operadores das ETE's, conforme exemplo abaixo:

Acompanhamento Hipoclorito

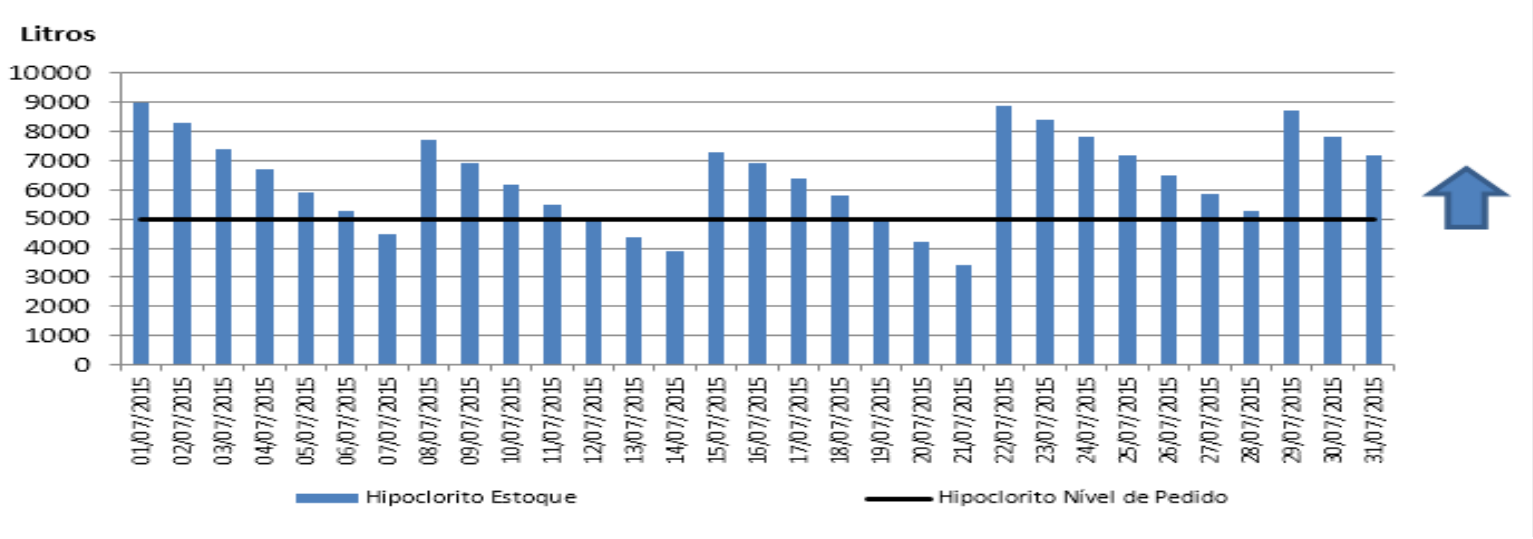

Figura 2: Gráfico de Acompanhamento de dosagem química. Fonte: ETE-Aços Longos

Também foi realizado um levantamento dos números de ocorrências de desvio de qualidade da água e interferências nos processos por falta dos produtos químicos, avaliação dos custos de transporte das entregas em emergências (pernoites e finais de semana) e multas ambientais por descumprimento de parâmetros para descartes no meio hídrico. 


\section{RESULTADOS E DISCUSSÃO}

Em relação às ocorrências de desvio de qualidade dos processos atendidos pela GDU, por falta de produtos químicos, foram constatados os seguintes números, segundo dados pesquisados no SIGMA:

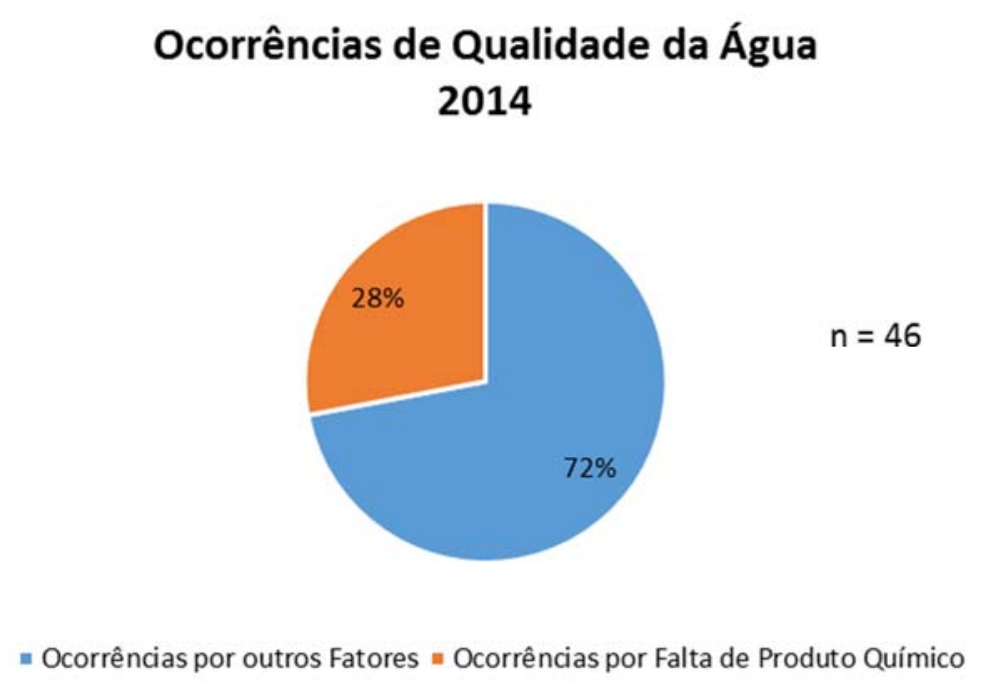

Figura 3: Gráfico de Ocorrência com Qualidade da Água. Fonte: Os autores - Pesquisa realizada no SIGMA

Abaixo, registros de ocorrência no SIGMA relativos a perda de parâmetros de qualidade da água por falta de produtos químicos:

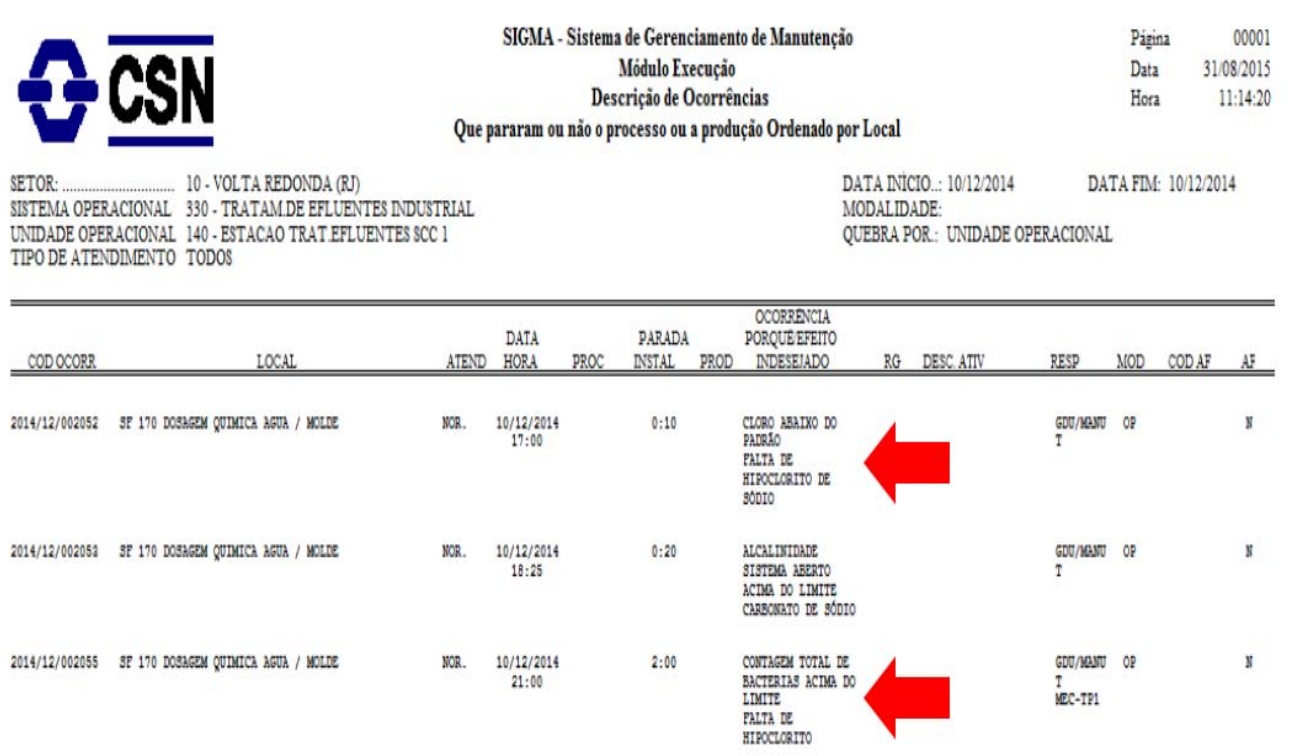

Figura 4: Relatório de Ocorrências no SIGMA. Fonte: SIGMA

Dentre essas ocorrências relacionando produtos químicos, nenhuma causou parada na produção, mas 12 ocorrências tiveram potencial para gerar algum dano ou interferência no cliente, a curto ou a longo prazo e 01 ocorrência afetou o meio ambiente (COA 266/2014 - ocorrência no SIGMA 2014/05/003973 - ETEO - OG acima de $20 \mathrm{mg} / \mathrm{L}$ ), totalizando em $28 \%$ de ocorrências significativas devido à falta de produtos químicos. 
As ocorrências analisadas e apuradas geraram evidencias de que isto ocorreu, a exemplo da ocorrência registrada no Sigma n² 2014/06/000525, com apontamento no diagrama de causa e efeito e árvore de causa, mostrados nas figuras 4 e 5, abaixo:

\section{RELACAO ENTRE GAUSA E EFETIO}

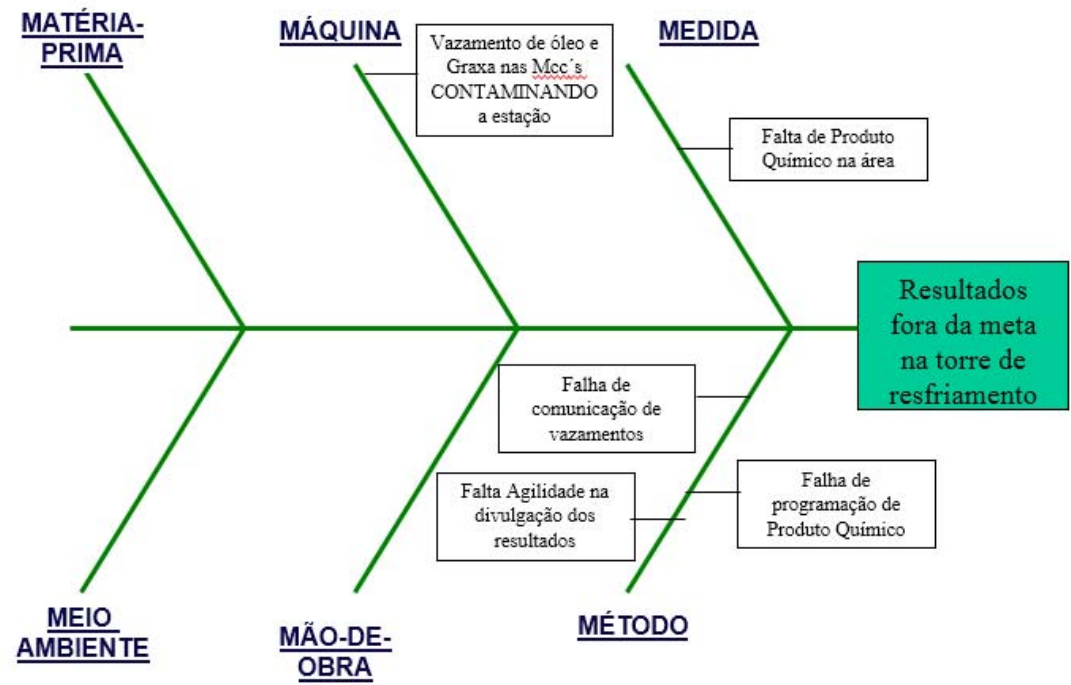

Figura 4: Diagrama de Causa e Efeito - FRAF 204/06/000525 - Elevados terores de SS - ETE-CC. Fonte: GDU

\section{NRVORE DE CAUSAS}

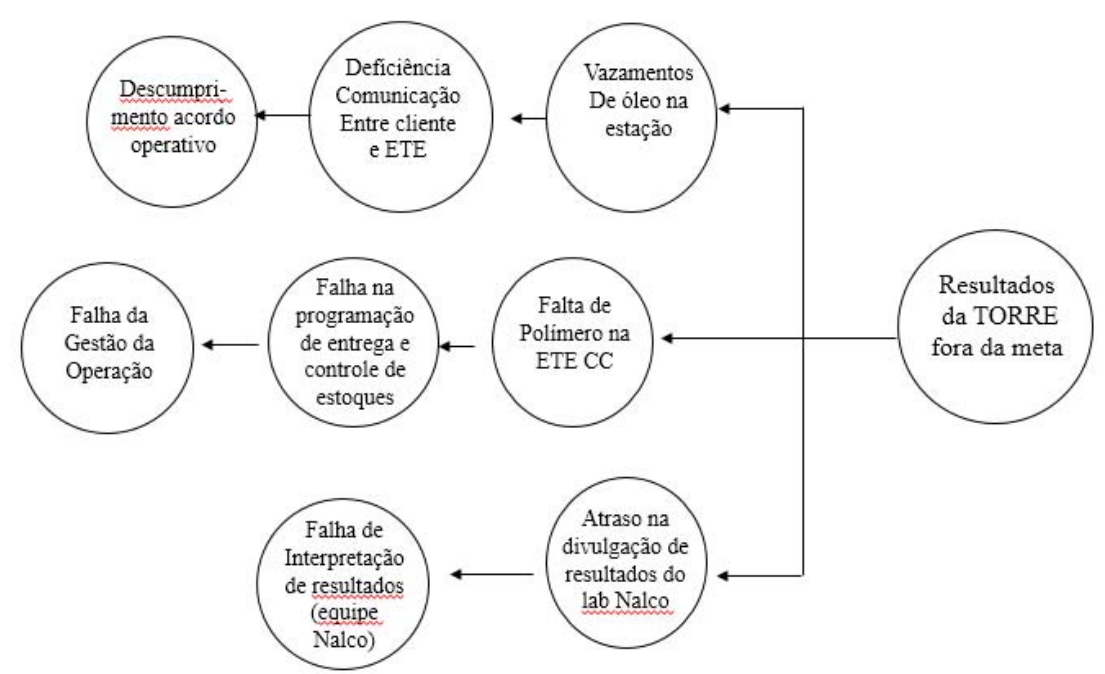

Figura 5: Árvore de Causa - FRAF 2014/06/000525 - Elevados teores de SS - ETE-CC. Fonte: GDU

Em 2015, após a implantação deste sistema de gerenciamento, não foram constatados problemas de falta de produtos químicos nas áreas.

Quanto ao custo, podemos observar que ao longo dos últimos anos, a GDU já vem realizando trabalhos de redução de custo, e que este trabalho complementa as ações anteriores, estando alinhado ao planejamento de redução de custos da empresa. Não foi possível mensurar quanto foi o impacto econômico após a implantação deste 
sistema, mas é importante destacar que o atual sistema de gerenciamento dos produtos químicos está focado com os resultados de qualidade da água e busca atender os consumos estabelecidos nos contratos firmados entre os fornecedores, evitando desperdícios.

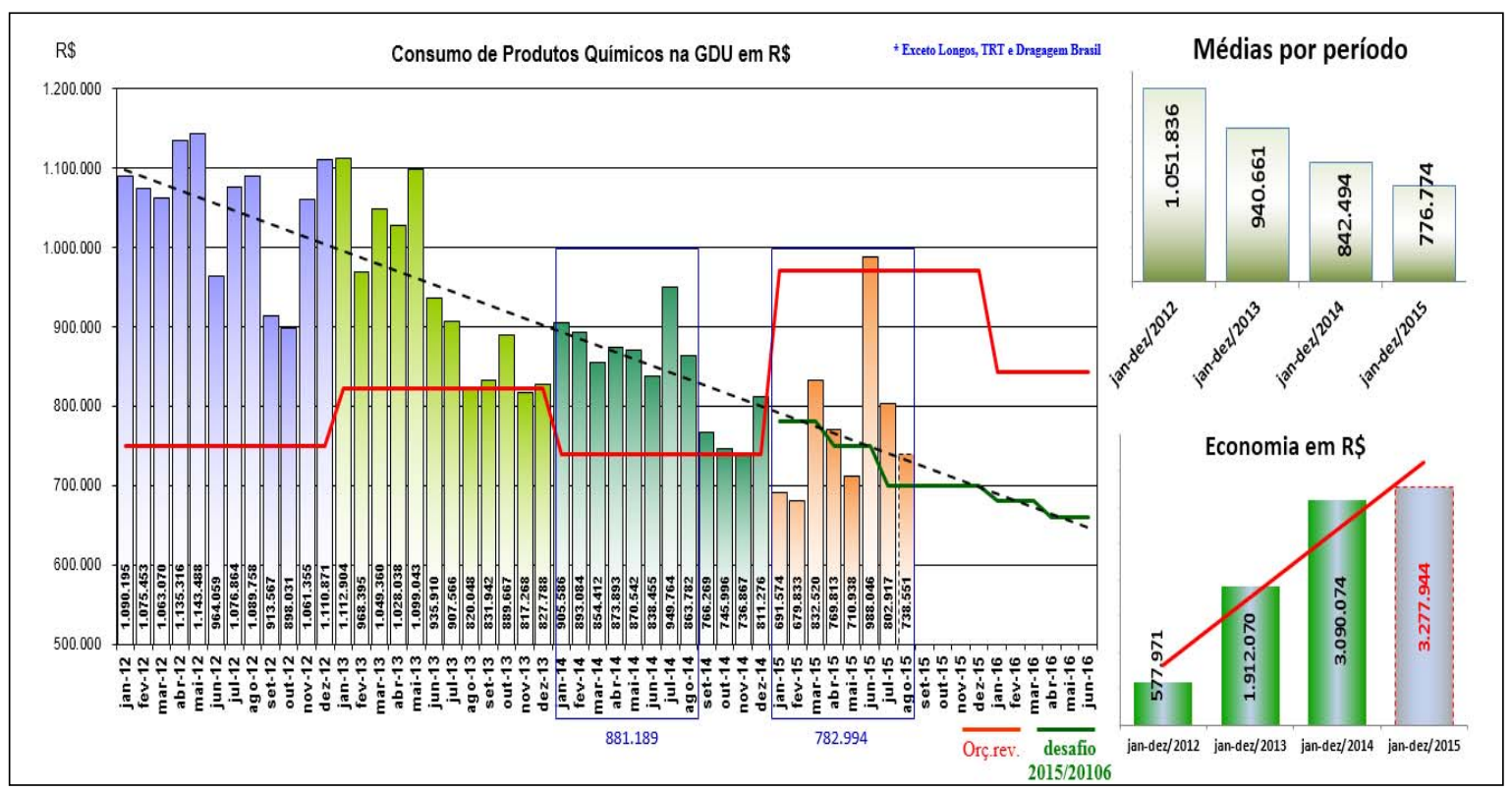

Figura 6: Gráfico do Consumo de Produtos Químicos na GDU em R\$. Fonte: GDU - dados extraídos do SAP

Podemos constatar que com as ações implantadas em conjunto na GDU, a previsão de economia com produtos químicos é crescente, e atinge a valores significativos, em torno de $\mathrm{R} \$ 3.277 .00$, projetados até Dezembro de 2015.

Outro ponto importante a ser considerado é sobre os custos evitados com pedidos em emergências, tais como os transportes para atendimento a em finais de semana, que resultam em pagamentos de diárias, pernoites e horas extras com pessoal próprio e contratado e multas ambientais por não atendimento a parâmetros ambientais, que podem chegar a $\mathrm{R} \$ 50.000 .000,00$.

Foi constatado que o número médio de entregas em emergências na GDU em 2014 foi de 1,58 por mês, sendo elas feitas aos finais de semana em meio expediente, e apresentaram o seguinte resultado (Tabela 1):

Tabela 1: Entregas em Emergência na GDU 2014

\begin{tabular}{|c|c|c|}
\hline Entregas em emergência na GDU - 2014 & Quantidade & Custo (R\$) \\
\hline Janeiro & 1 & 350 \\
\hline Fevereiro & 2 & 700 \\
\hline Março & 2 & 700 \\
\hline Abril & 1 & 350 \\
\hline Maio & 2 & 700 \\
\hline Junho & 1 & 350 \\
\hline Julho & 2 & 700 \\
\hline Agosto & 1 & 350 \\
\hline Setembro & 2 & 700 \\
\hline Outubro & 1 & 350 \\
\hline Novembro & 2 & 700 \\
\hline Dezembro & 2 & 700 \\
\hline Média & 1,58 & 553 \\
\hline Total & 19 & 6650 \\
\hline
\end{tabular}


Com base em dados de 2014, podemos concluir que em 2015 estaremos evitando um custo de $\mathrm{R} \$ 6650,00$ com diárias/pernoites de caminhões para entregas em emergência. Com esta verba, podemos comprar 6 containers de polímero, um dos produtos químicos mais utilizados na GDU, por exemplo.

Um ganho indireto que foi obtido com este trabalho foi a medição de consumo dos produtos químicos, que anteriormente não era acompanhada, e que contribuiu para a redução de custo, conforme gráfico da figura 6 , já citado acima.

\section{CONCLUSÃO}

O trabalho complementa o que já existe na GDU em relação às ações de redução de custo com produtos químicos e ajudou a manter sob controle e tornar previsível os consumos, datas de realizações de pedidos e de entregas dos produtos químicos, evitando desperdícios e descontroles dos parâmetros de qualidade da água, que poderiam demandar em custos com transportes extras e multas ambientais. Aumentou a assertividade e o comprometimento da equipe com os processos, e aliado a isso tudo, vem gerando uma economia significativa. 